\title{
Delamination tolerance studies in laminated composite panels
}

\author{
$\mathrm{K}^{\mathrm{L}}$ SINGH $^{1}$, B DATTAGURU ${ }^{1}$, T S RAMAMURTHY ${ }^{1}$ AND \\ P D MANGALGIRI ${ }^{2}$
}

${ }^{1}$ Department of Aerospace Engineering, Indian Institute of Science, Bangalore 560012 , India

${ }^{2}$ Aeronautical Development Agency, PB No. 1718, Vimanapura Post, Bangalore 560017, India

e-mail: datgur@aero.iisc.ernet.in

\begin{abstract}
Determination of levels of tolerance in delaminated composite panels is an important issue in composite structures technology. The primary intention is to analyse delaminated composite panels and estimate Strain Energy Release Rate (SERR) parameters at the delamination front to feed into acceptability criteria. Large deformation analysis is necessary to cater for excessive rotational deformations in the delaminated sublaminate. Modified Virtual Crack Closure Integral (MVCCI) is used to estimate all the three SERR components at the delamination front from the finite element output containing displacements, strains and stresses. The applied loading conditions are particularly critical and compressive loading on the panel could lead to buckling of the delaminated sublaminate and consequent growth of delamination. Numerical results are presented for circular delamination of various sizes and delamination at various interfaces (varying depth-wise location) between the base- and the sub-laminates. Numerical data are also presented on the effect of bi-axial loading and in particular on compressive loading in both directions. The results can be used to estimate delamination tolerance at various depths (or at various interfaces) in the laminate.
\end{abstract}

Keywords. Delamination, modified virtual crack closure integral; strain energy release rate.

\section{Introduction}

Delaminations (figure 1) occur in composite structures, and these can be broadly classified as material induced (due to poor quality of raw material or excessive solvent in resin), process induced (because of non-wetting of fibres or resin starvation), thermally induced (owing to non-uniform temperature rise in laminate resulting in thermal stresses or improper curing), and stress/environment induced (due to low velocity impact loading or hostile environment such as high humidity, vibration etc.). These defects do not necessarily manifest themselves on the outer surface of the component, could grow during operation and may lead to total delamination of some of the layers of the component. The 


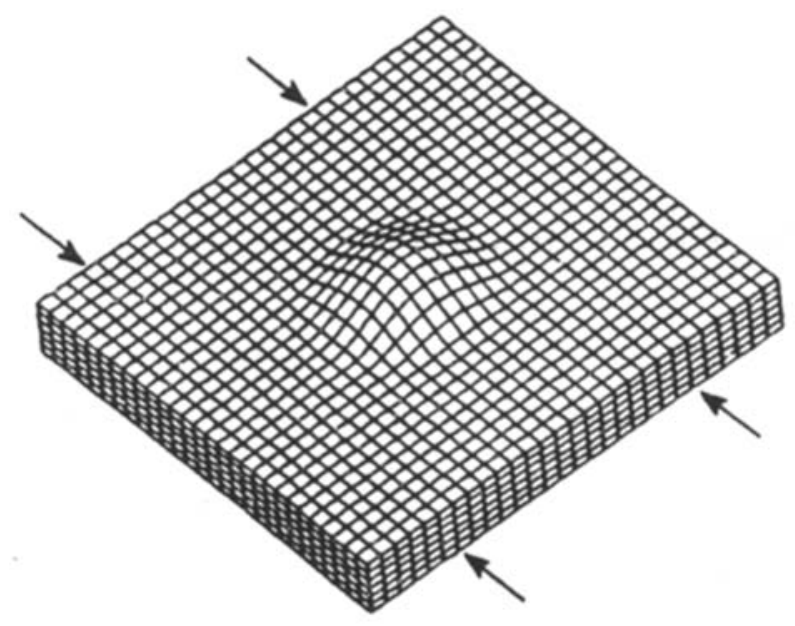

Figure 1. Typical configuration of a panel with embedded delamination.

consequences of this delamination are loss of stiffness and critical compressive strength of the laminated panel.

Three-dimensional finite element software was developed to analyse circular/elliptical delaminations and to assess tolerance levels based on fracture mechanics principles. Special features of the analysis include the adoption of a super-element (Jones et al 1984) and its implementation to handle large numbers of layers in the composite, the use of appropriate reduced integration (Naganarayana 1991) techniques, and the implementation of recent developments (Badari Narayan et al 1994) by some of the present authors to estimate Strain Energy Release Rate (SERR) components at curved three-dimensional delamination front. Parametric study was conducted on a typical $\pi / 4$ laminate of 42 layers. Numerical studies were conducted to bring out the influence of delamination size and depthwise location, and effect of uni-axial and bi-axial compressive loads on the panel.

\section{Description of the problem}

A square composite panel of $2 \mathrm{~W} \times 2 \mathrm{~W}$ containing an elliptic delamination of (figures 1 and 2) $2 \mathrm{a} \times 2 \mathrm{~b}$ located at the centre of the panel is considered for analysis. $N_{s}$ specify depth-wise locations of delamination interfaces. The panel is simply supported along all the four edges. For cases of uni-axial or bi-axial loading, only one quarter of the panel (ABCD) is analysed. The loading is compressive strain $\varepsilon_{x}$ and $\varepsilon_{y}$ along $x$ and $y$ directions applied along $\mathrm{BC} \& \mathrm{CD}$. $\mathrm{AB} \& \mathrm{AD}$ are assumed to be axes of symmetry. In principle, though the geometry of the panel is symmetric about $\mathrm{AB}$ and $\mathrm{AD}$, the $+45^{0}-45^{0}$ layers would disturb symmetry. However, numerical studies conducted in the present work (not presented in this paper for the sake of brevity) showed that the assumption of symmetry would lead to insignificant errors as long as the laminate is balanced.

\subsection{Mechanical properties of the unidirectional tape}

$$
\begin{array}{lll}
E_{11}=130000 \mathrm{MPa} & G_{23}=3750 \mathrm{MPa} & \nu_{23}=0.45 \\
E_{22}=10000 \mathrm{MPa} & G_{13}=5000 \mathrm{MPa} & \nu_{13}=0.35 \\
E_{33}=10000 \mathrm{MPa} & G_{12}=5000 \mathrm{MPa} & \nu_{12}=0.35
\end{array}
$$



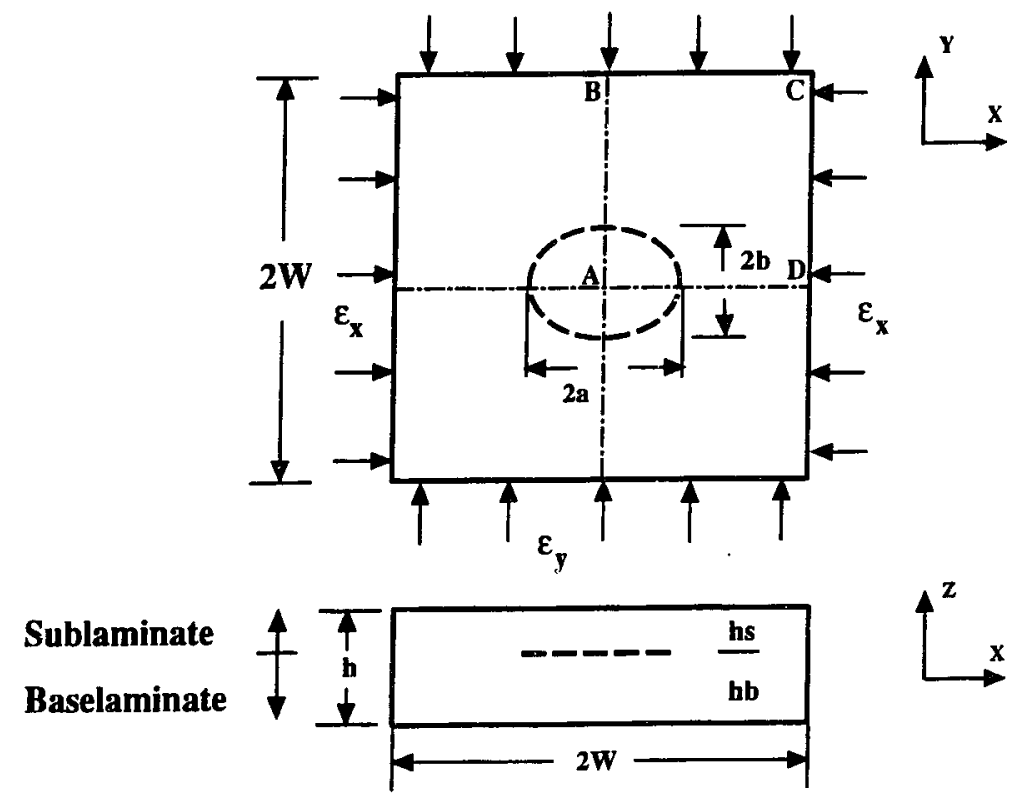

Figure 2. Nomenclature of the panel analysed.

1: Fibre direction

2: Transverse direction (in the plane of the laminate)

3: Thickness direction.

\section{Special features of the analysis}

\subsection{Super-element}

In order to keep the degrees of freedom of the finite element model within a reasonable number and to keep the model to a realistic size a super-element concept developed by Jones et al (1984) is used. The derivation is presented here, since there were some mistakes in the earlier work (Jones et al 1984), though the final equations presented there are correct. In this case, a super-element contains more than one composite lamina with fibres oriented in different directions in each of them. So it is a stack of orthotropic layers of the composite within the domain of each super-element. Consider the development of a super-element with ' $N$ ' layers (figure 3). The stiffness matrix for this super-element is obtained by integrating in the volume of each layer and summing up over the $N$ layers. This is given by

$$
\begin{aligned}
K & =\iint_{v} \int B^{T} D B \mathrm{~d} v \\
& =\sum_{i=1}^{N} \iint_{v_{l}} \int B^{T} D_{i} B \mathbf{d} v_{i} .
\end{aligned}
$$

Using three-point interpolation function for the $Z$ coordinate, one can write

$$
Z=-Z_{1}\{(1-\xi) \xi\} / 2+Z_{2}\left(1-\xi^{2}\right)+Z_{3}\{(1+\xi) \xi\} / 2 .
$$




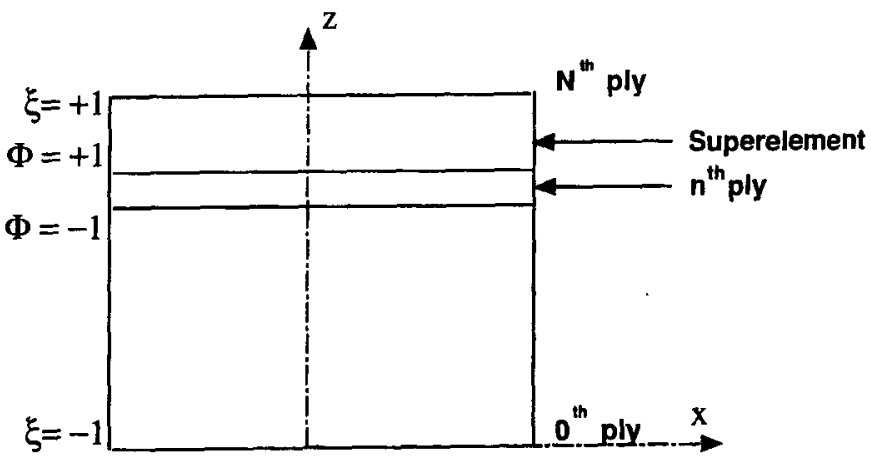

Figure 3. Internal coordinate system for superelement.

Substituting into the condition

$$
\begin{aligned}
& \text { at } \quad \xi=-1, \quad Z=0, \\
& \text { at } \xi=0, \quad Z=N h_{0} / 2, \\
& \text { at } \xi=1, \quad Z=N h_{0},
\end{aligned}
$$

results in $Z_{1}=0, Z_{2}=N h_{0} / 2$ and $Z_{3}=N h_{0}$, where $N$ is the total number of layers in the super-element and $h_{0}$ is the thickness of each layer. With this, the above expression becomes,

$$
Z=N h_{0}(1+\xi) / 2
$$

$\phi$ varying between -1 and 1 is the natural coordinate system for the $n$th ply and $\phi$ is related to $Z$ by the expression,

$$
Z / h_{0}=(\phi+1) / 2+\left(P_{n}-1\right)
$$

where the plies are assigned the number $P_{n} ; P_{n}=1$ being the bottom ply and $P_{n}=N$ being the top ply of the superelement. Using (3) and (4), $\phi$ can be related to $\xi$ by the expression,

$$
\xi=\left(\phi+2 P_{n}-1-N\right) / N .
$$

equation (5) is made use of while calculating the stiffness matrix, $K=\iiint B^{T} D B \mathrm{~d} v . A$ 2-point Gaussian quadrature is generally used in each ply and the coordinates of any point in the $n$th ply in the $\phi$ co-ordinate system is transformed into the $\xi$ coordinate system using (5). The corresponding weighting factors in the $\xi$ co-ordinate system are then $1 / N$ times the weighting factors in the $\phi$ co-ordinate system.

Through the use of super-elements, it is possible to accurately account for the variation of properties due to varying fibre orientations from lamina to lamina within each superelement in evaluating the stiffness matrix of various elements.

It is well-known in the literature that an eight-node brick element is poor in bending. One of the methods of overcoming this is by the use of reduced integration for shear strains.

The lamina is in the $x-y$ plane and the use of full integration for $\tau_{x y}$ is recommended (Naganarayana 1991). Based on this, in the current work one-point integration is used only for $\tau_{y z}$ and $\tau_{z x}$. A similar approach is adopted for nonlinear terms in geometric nonlinearity. Further, for the nonlinear component $\left(B_{L}\right) 2 \times 1 \times 2$ integration is adopted for four components of strains (direct strains and $\tau_{x y}$ ). This reduced integration is checked on several examples of beams and plates. 


\subsection{Geometric nonlinear analysis}

When the delaminated panel is subjected to compressive loading, there is buckling of delaminated sublaminate at some small load (small with reference to the buckling load of the total panel). Beyond this load level there is increased deformation in this region, which is likely to be beyond the small deflection theory (Whitcomb 1984, 1988). To handle this situation, the configuration is analysed by large deformation analysis. An incremental iterative approach is followed (Zienckiwicz \& Taylor 1991). In each incremental load step, using Lagrangian approach the nonlinear strain displacement relations given below are used

$$
\begin{aligned}
\varepsilon_{x} & =\partial u / \partial x+1 / 2\left[(\partial u / \partial x)^{2}+(\partial v / \partial x)^{2}+(\partial w / \partial x)^{2}\right] \\
\varepsilon_{y} & =\partial v / \partial y+1 / 2\left[(\partial u / \partial y)^{2}+(\partial v / \partial y)^{2}+(\partial w / \partial y)^{2}\right] \\
\gamma_{x y} & =\partial u / \partial y+\partial v / \partial x+[\partial u / \partial x \partial u / \partial y+\partial v / \partial x \partial v / \partial y+\partial w / \partial x \partial w / \partial y]
\end{aligned}
$$

This results in the $[\mathrm{B}]$ matrix relating strains to nodal displacement, which can be expressed as

$$
B=B_{0}+B_{L},
$$

where $B_{0}$ is the linear part and $B_{L}$ is the nonlinear part, which is a function of displacements and their derivatives. The total tangent stiffness matrix $K_{T}=K_{0}+K_{L}+K_{\sigma}$, where $K_{\sigma}$ is the initial stress matrix evaluated as given by Zienckiwicz \& Taylor (1991), and the resulting $[K]$ matrix is nonlinear. The solution of this nonlinear equation is carried out by an iterative procedure with modified Newton-Raphson method (Zienckiwicz \& Taylor 1991). In this method, the tangent stiffness matrix is updated only after a fixed number of iterations. In this present work it is updated after four iterations.

At the end of the convergent solution for every load step, the geometry is updated, and analysis is carried out for the next load step. Such an updated Lagrangian approach is expected to provide faster convergence for every load step.

\subsection{MVCCI for general hexahedral element}

The Strain Energy Release Rate (SERR) components are obtained using Modified Virtual Crack Closure Integral (MVCCI) (Rybicki \& Kanninen 1977; Buchholz 1984; Raju 1986; Badari Narayan et al 1994). MVCCI equations for the three components $G_{\mathrm{I}}, G_{\mathrm{II}}, G_{\mathrm{III}}$ are element-dependent and these have to be derived for the general hexahedral element. Consider a curved delamination front (figure 4). A local coordinate system $n, t, z$ is used to identify three modes of failure.

Delamination is in the $x-y$ (or $n-t)$ plane (figure 4). The expression for $G_{I}$ based on MVCCI is expressed in the corresponding natural coordinate system as

$$
G_{\mathrm{I}}=1 /\left(2 \Delta A_{i}\right) \int_{\Delta A_{i}} \sigma_{z}(\xi, \eta) w(\xi, \eta)|J| \mathrm{d} \xi \mathrm{d} \eta
$$

where $\Delta A_{i}$ is the area of the element where MVCCI is being estimated. The area of the element ahead of delamination is $\Delta A_{r}$ and behind is $\Delta A_{l}$. Assuming a small virtual delamination extension and a refined mesh, $\Delta A_{l} \cong \Delta A_{r} \cong \Delta A_{i}$. Based on the well-referred single analysis, the stress distribution is picked up from the element ahead of the delamination 


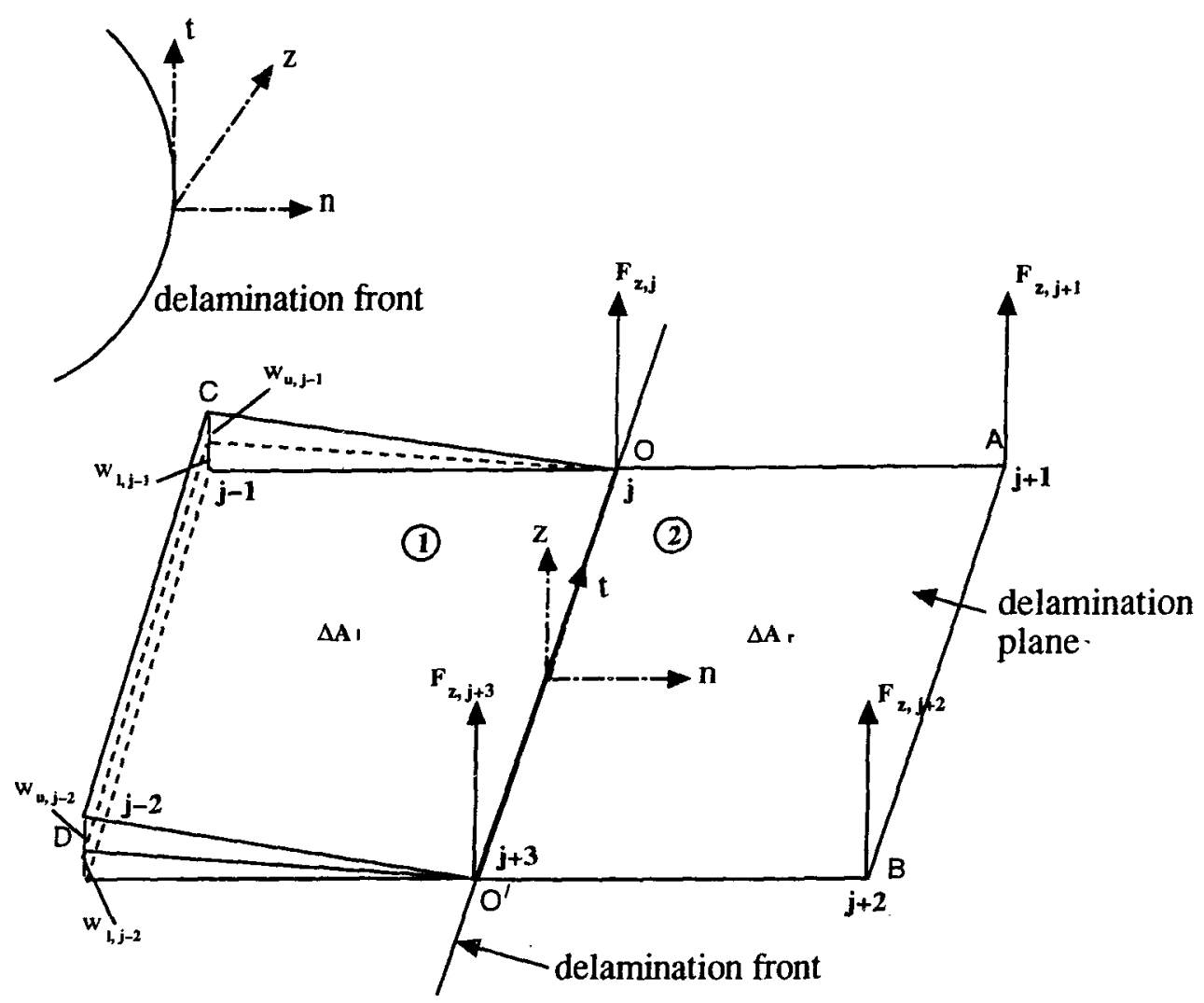

Figure 4. Nodal displacements and forces in the plane of delamination: 8-node brick element.

front $\left(\mathrm{OABO}^{\prime}\right)$ and the displacement distribution from the element behind the delamination front $\left(O^{\prime} D C O\right)$, and their product is integrated over one element area to estimate SERR components. Here $G_{\mathrm{I}}$ is estimated by assuming an area of virtual delamination extension and estimating the work to be done to close the delamination back to original size. The normal stress $\sigma_{z}$ distribution in the element $\mathrm{OABO}^{\prime}$ ahead of crack front is expressed as a polynomial in $\xi, \eta$ as

$$
\sigma_{z}(\xi, \eta)=b_{0}+b_{1} \xi+b_{2} \eta+b_{3} \xi \eta \text {. }
$$

The constants $b_{0}, b_{1}, b_{2}, b_{3}$ can be expressed as consistent load vectors and can be expressed in terms of nodal forces $F_{z, j}, F_{z, j+1}, F_{z, j+2}, F_{z, j+3}$. The crack-opening displacements (in the element $\mathrm{O}^{\prime} \mathrm{DCO}$ behind the crack front) are also expressed as a polynomial in $\xi, \eta$ as

$$
w(\xi, \eta)=a_{0}+a_{1} \xi+a_{2} \eta+a_{3} \xi \eta
$$

The constants $a_{0}, a_{1}, a_{2}, a_{3}$ can be related to nodal displacements. For mode I they are expressed in terms of delamination opening displacements $W_{j-1}$ and $W_{j-2}$ at the nodes $(j-1)$ and $(j-2)$.

In addition, the Jacobian of the transformation can be expressed as,

$$
|J|=J_{0}+J_{1} \xi+J_{2} \eta
$$


The SERR expression can be derived as

$$
\begin{aligned}
G_{\mathrm{I}}= & \left(1 / 2 \Delta A_{i}\right)\left[4 J_{0}\left(a_{0} b_{0}+a_{1} b_{1} / 3+a_{2} b_{2} / 3+a_{3} b_{3} / 9\right)\right. \\
& +4 J_{1}\left(a_{0} b_{1} / 3+a_{1} b_{0} / 3+a_{2} b_{3} / 9+a_{3} b_{2} / 9\right) \\
& \left.+4 J_{2}\left(a_{0} b_{2} / 3+a_{1} b_{3} / 9+a_{2} b_{0} / 3+a_{3} b_{1} / 9\right)\right] .
\end{aligned}
$$

If the element face $\mathrm{OABO}^{\prime}$ in figure 4 is rectangular, $J_{1}=J_{2}=0$, and after considerable simplification, it can be shown that

$$
G_{\mathrm{I}}=1 /\left(2 \Delta A_{i}\right)\left[F_{z, j} w_{j-1}+F_{z,(j+3)} w_{j-2}\right] .
$$

By repeating the above procedure for different segments of delamination front, one obtains the variation of G's along the crack front. Similar expressions can be derived for second and third modes of SERR. For mode-II $\tau_{n z}, u_{n}$ replace $\sigma_{z}$ and $w$ and for mode-III $\tau_{n t}, u_{t}$ replace $\sigma_{z}$ and $w$ in (8) and the corresponding expressions can be derived.

The delamination is between two orthotropic layers, which can have fibres running in different orientations. It is essentially an interface crack (delamination) between two dissimilar materials. It is known (Williams 1959) that such a situation could lead to complex singularity and there are difficulties in achieving convergence for strain energy release rate components as virtual delamination extension size $\Delta A_{i} \rightarrow 0$. A study on this was carried by Raju, who showed that reliable estimate of $G_{I}, G_{\mathrm{II}}, G_{\mathrm{III}}$ could be obtained when virtual delamination extension (i.e., length perpendicular to the front) is of the order of the lamina thickness. The same is followed in the current FE model.

\section{FE model}

Figure 5 shows a typical finite element model. The elements are eight-node iso-parametric hexahedrons. The FE model was created by specifying the number of radial divisions (graded not equal) $N R$ and the number of equal divisions in the theta directions $N T H$. After a systematic convergence study, numerical computations were carried out on a finite

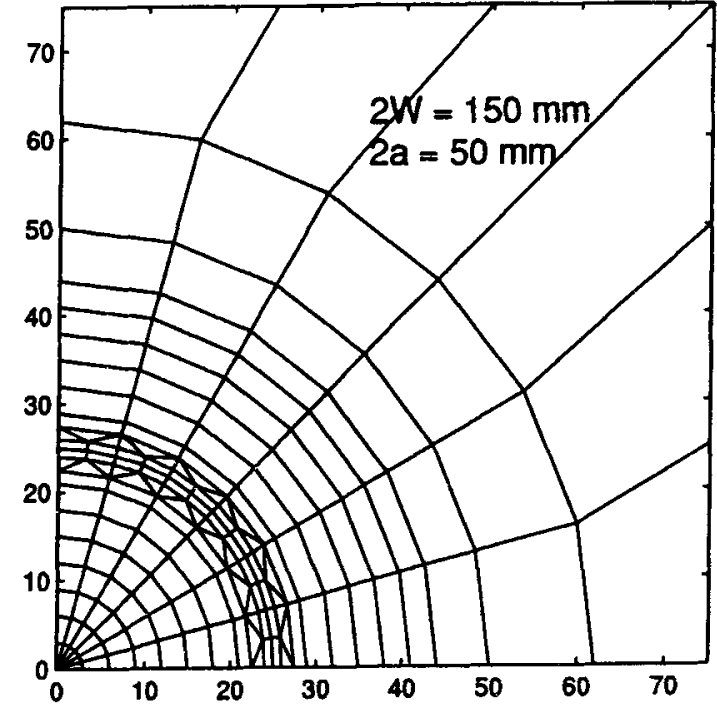

Figure 5. FE mesh in the plane of circular delamination $N N=1896, N E=1620, N R=$ $20, N T H=12$. 
element model with $N R=20$ and $N T H=12$ (7.5 divisions). The maximum number of superelements used across the thickness was 10 and nearly 6000 degrees of freedom were employed in all the cases. Because of symmetry it is sufficient to model only one-fourth of the specimen. The displacement boundary conditions are $u=0$ on $x=0 ; v=0$ on $y=0$; $w=0$ on $z=0$ along the edges $(x=W$ and $y=W$ ). The last boundary condition implies simply supported edges all round the panel.

The loading on the panel is applied as a constant strain along the outer edges. For uniaxial compressive loading constant displacement $\left(W \varepsilon_{x}\right)$ is applied along the edge $x=W$ where $\varepsilon_{x}$ is the uni-axial compressive strain. For bi-axial loading with specified $\varepsilon_{\boldsymbol{x}}, \varepsilon_{y}$ constant displacements are applied along edges $x=W$ and $y=W$. The net loading in each direction is obtained by summing up the reactions at the end of FE analysis.

\section{Results}

The software developed was checked by solving the classical problem of a penny-shaped crack in a large isotropic solid subjected to far-field tensile loads. The results of this are not presented here, but they compare within one percent of the earlier solutions (Broek 1978).

De Georges et al (1992) have conducted experimental investigations till failure and corresponding finite element analysis for a 24-layer composite with central delamination. The current software is used to analyse the same configuration. The deflection at the centre of the delamination from the experimental, and numerical results of De Georges et al (1992) are compared with the present FEM work and good agreement is seen up to onset of buckling strength (figure 6 ).

A parametric study was conducted on a 42-layer laminate whose sequence is as follows: [323223314322141414141] s, where $1,2,3,4$ correspond to $0^{\circ}, 45^{\circ},-45^{\circ}, 90^{\circ}$ respectively. The laminate is of dimension $150 \times 150 \mathrm{~mm}$ and of $6.3 \mathrm{~mm}$ thickness. As discussed earlier, only a quarter of the panel was analysed.

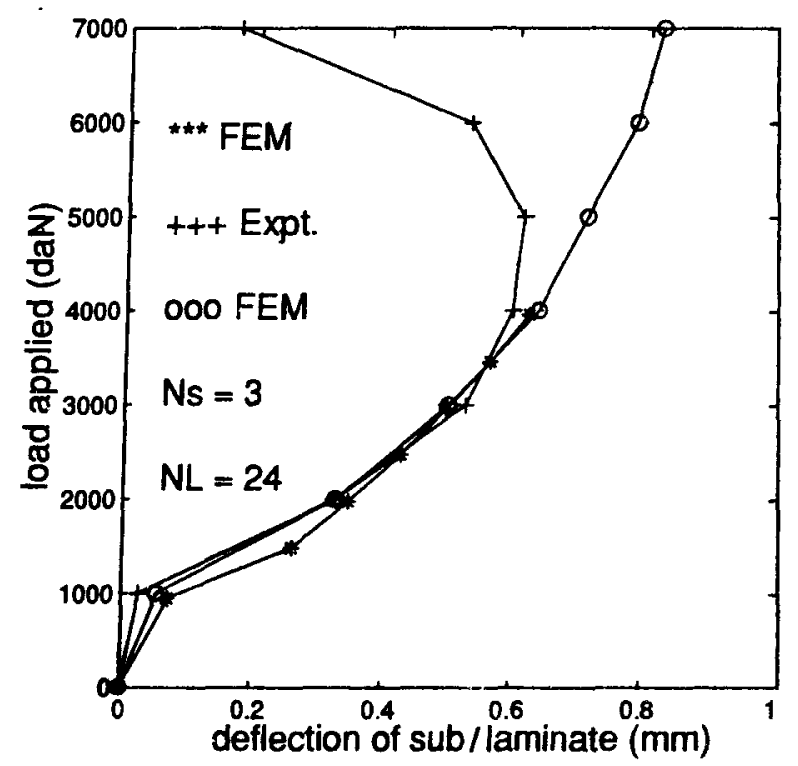

Figure 6. Comparison of sub-laminate deflection vs load (De Georges et al 1992). 


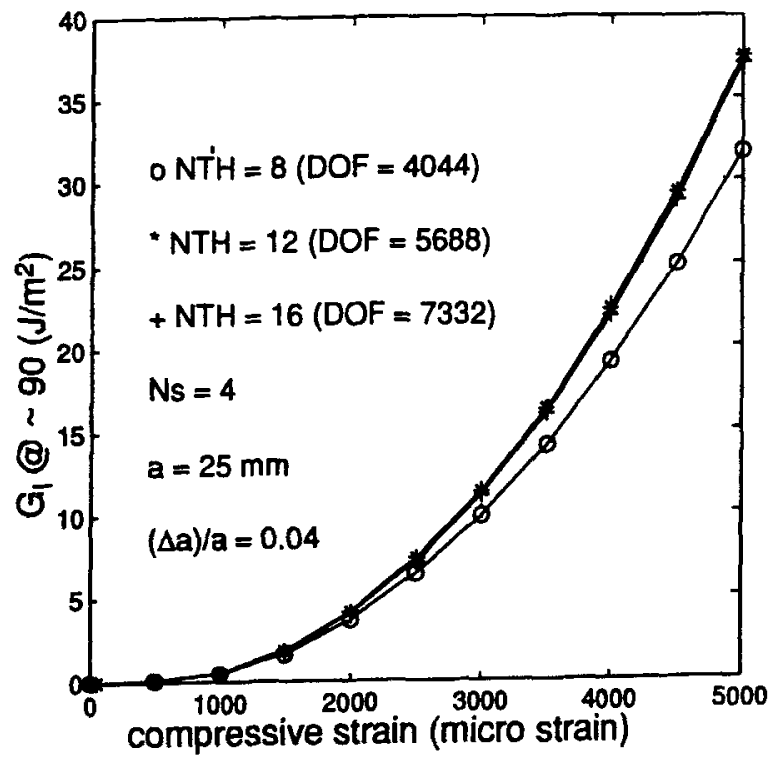

Figure 7. Convergence of $G_{\mathrm{I}}$ with compressive strain.

\subsection{Convergence study}

The problem is modelled with three levels of mesh refinement of 4044, 5688 and 7332 degrees of freedom representing the number of divisions along the crack front as 8,12 and 16 respectively. It is seen in figure 7 the variation of $G_{I}$ at $90^{\circ}$ location for a circular delamination of $50 \mathrm{~mm}$ diameter and located at the 4th interface, with the applied strain. The curves for the 2nd and 3rd models are very close and hence all further studies are carried out with 12 divisions along the crack front. It is seen from figure 8 that the

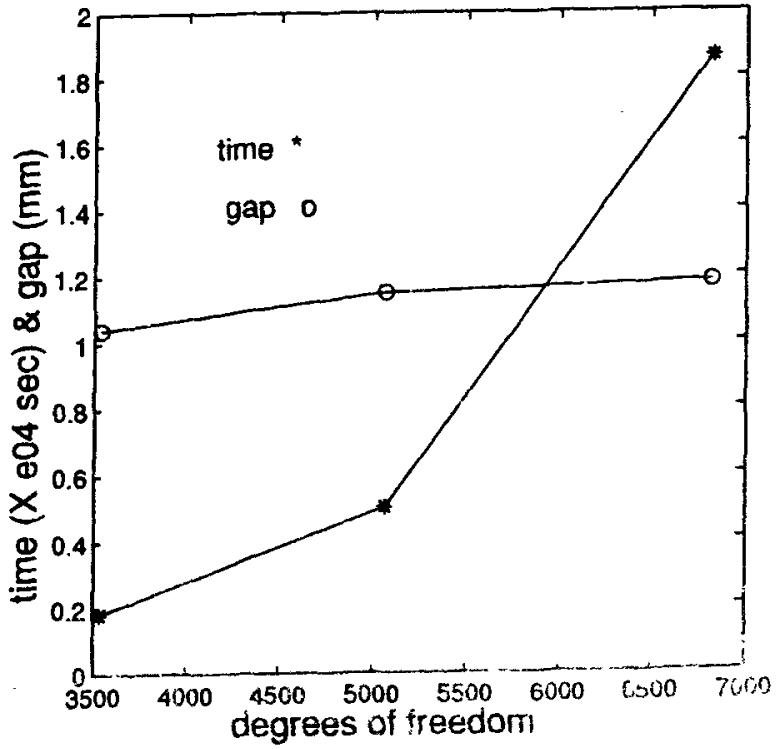

Nigure 8. Computational time for various. mes reintments and comver gence of comal gat whin dest" 


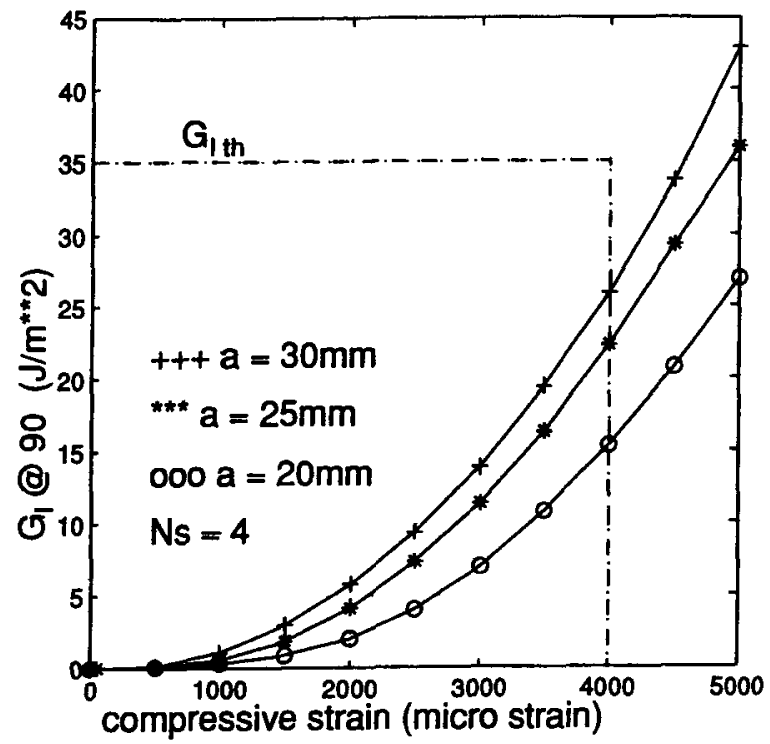

Figure 9. Effect of size of circular delamination on $G_{I}$.

computer time required beyond 5000 degrees does not warrant the accuracy achieved in the solution.

\subsection{Delamination tolerance studies: Effect of size, location and bi-axial loading}

Effect of the size of circular delamination located on the fourth interface on mode-I SERR is presented in figure 9. Obviously, the larger the delamination, the higher the mode-I SERR component. If $G_{\mathrm{I}}$ threshold $=35 \mathrm{~J} / \mathrm{m}^{2}$, it is seen that $60 \mathrm{~mm}$ diameter delamination could be tolerated at $4000 \mu$ strain. This can be suitably used in choosing the mean and alternate

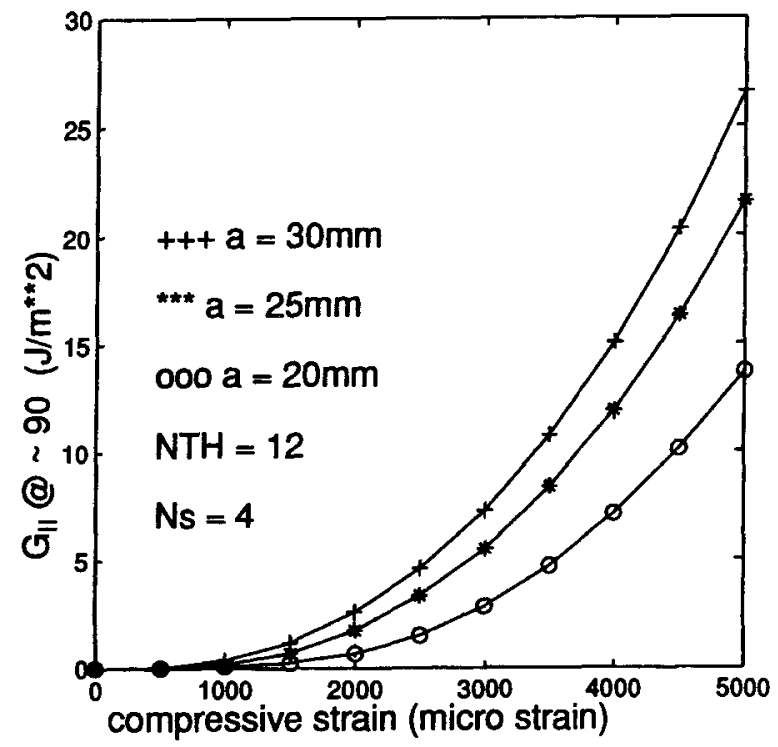

Figure 10. Effect of size of circular delamination on $G_{\mathrm{II}}$. 


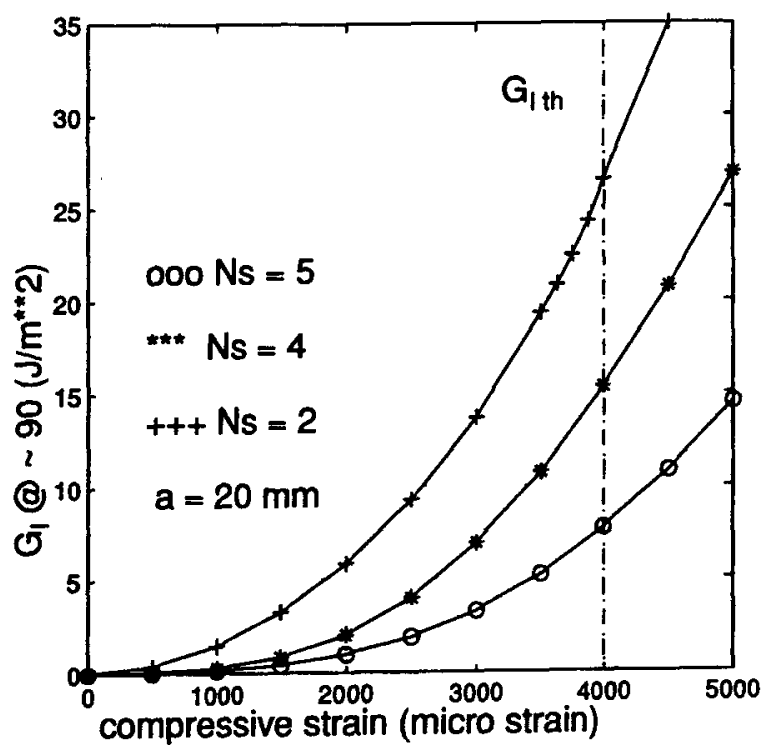

Figure 11. Effect of depthwise location of circular delamination on $G_{\text {I }}$.

components of fatigue loading. Figure 10 shows the variation of $G_{I I}$ due to a circular delamination at the fourth interface. It also presents the effect of the size of the delamination. It is seen that for a given applied compression the magnitude of $G_{\mathrm{II}}$ is always lower. It is also noted that $G_{\mathrm{III}}$ is insignificant compared to $G_{\mathrm{I}}, G_{\mathrm{II}}$ and hence is not presented.

The effect of location of circular delamination at different interfaces is as shown in figure 11. It is seen here that $40 \mathrm{~mm}$ diameter delamination can be tolerated at interfaces $N_{s} \geq 2$ up to $4000 \mu$ strains. Figure 12 shows the functional curve fit for strain parameter. It indicates that $G_{\max }$ is function of $\varepsilon^{3}$ for delamination at interfaces beyond the fourth $\left(N_{s} \geq 4\right)$.

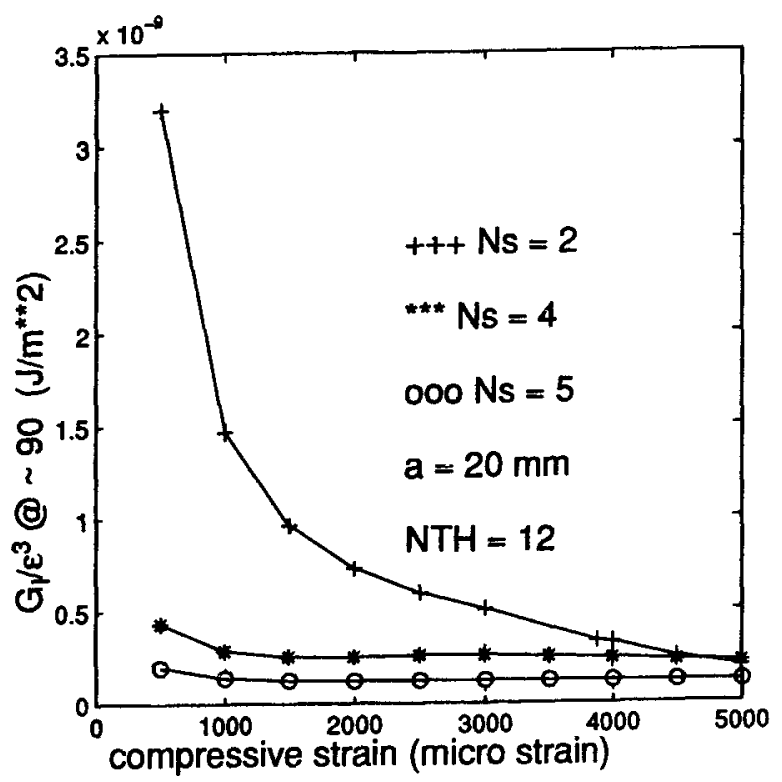

Figure 12. Variation of $G_{\mathrm{I}} / \varepsilon^{3}$ with compressive strain. 


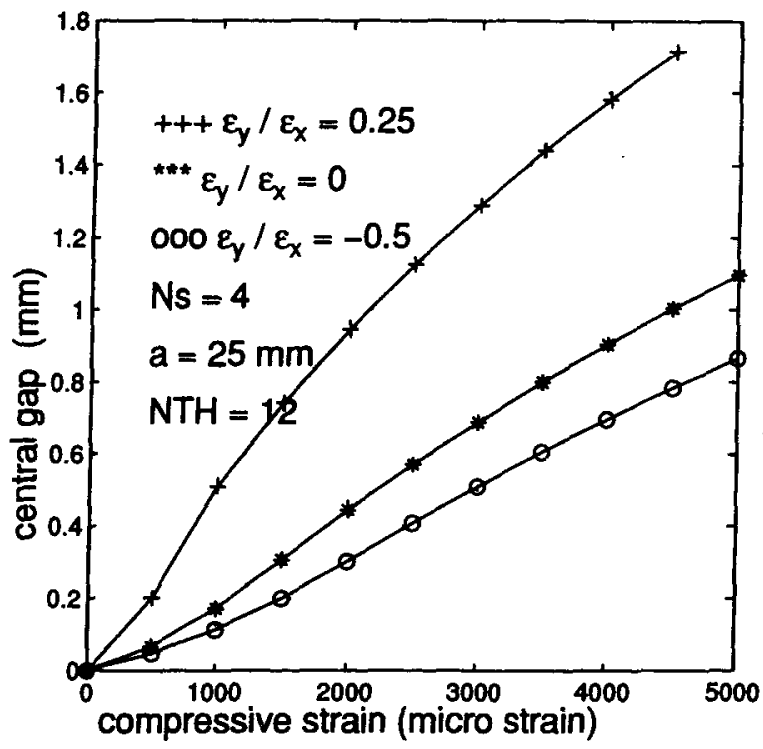

Figure 13. Effect of biaxial loading on central gap between base and sublaminate.

A laminate with a circular delamination of $50 \mathrm{~mm}$ was subjected to bi-axial strain system $\varepsilon_{x}, \varepsilon_{y}$. The transverse strain $\varepsilon_{y}$ is taken to be a constant proportion of $\varepsilon_{x}$. Three cases of loading are considered: (i) $\varepsilon_{y}=0.25 \varepsilon_{x}$, (ii) $\varepsilon_{y}=0.0$, and (ii) $\varepsilon_{y}=-0.5 \varepsilon_{x}$. Figure 13 presents the variation of central gap between base and sub-laminates with $\varepsilon_{x}$ for different $\varepsilon_{y} / \varepsilon_{x}$ values. As expected from the figure, this gap for bi-axial compression strain case (i) is greater compared to the other two cases. Figure 14 presents the variation of $G_{\mathrm{I}}$ along the delamination front for $\varepsilon_{x}=4000 \mu \varepsilon$ and various values of $\varepsilon_{y} / \varepsilon_{x}$. It is seen that maximum $G_{I}$ occurs at $90^{\circ}$ location for all the cases. Figure 15 presents $G_{I}$ at $90^{\circ}$ location with compressive strain $\varepsilon_{x}$ for various values of $\varepsilon_{y} / \varepsilon_{x}$. Here, it is critical for the case of bi-axial

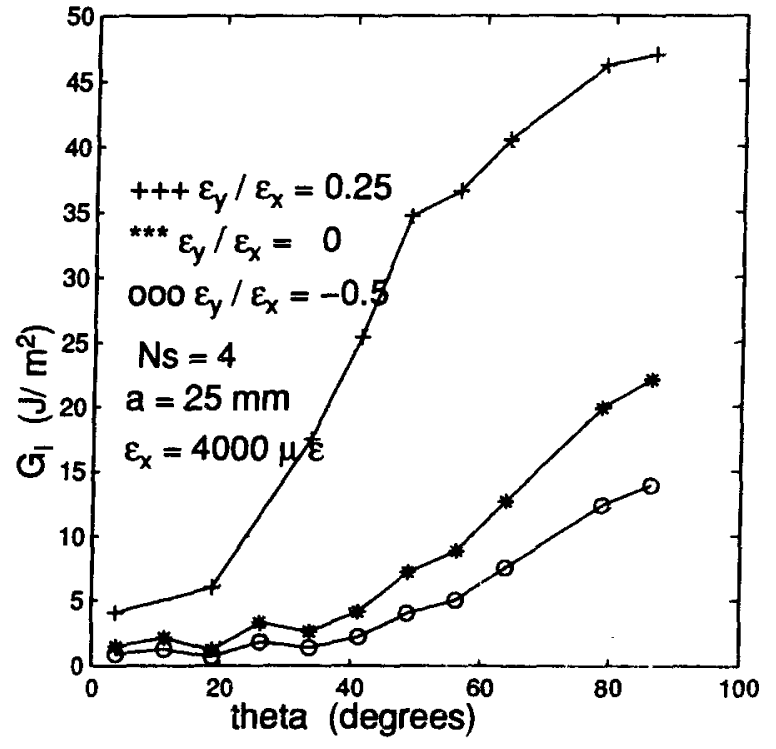

Figure 14. Effect of biaxial loading: variation of $G_{1}$ along the delamination front. 


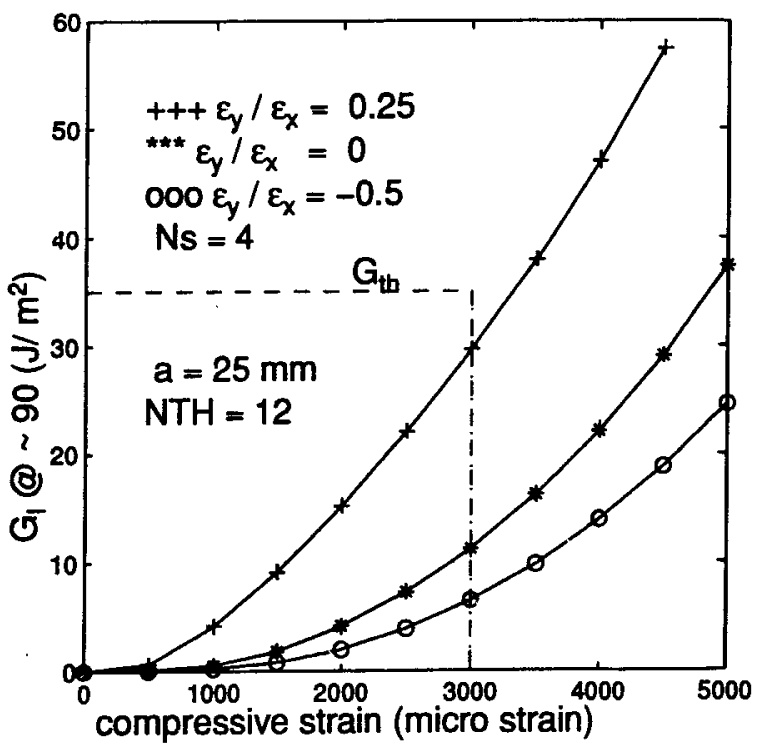

Figure 15. Effect of biaxial loading: Variation of $G_{I}$ with compressive strain.

compression strain (in both $x$ and $y$ directions) and in such cases delamination of size less than $50 \mathrm{~mm}$ diameter can only be tolerated.

\section{Conclusions}

Three-dimensional finite element analysis for a delaminated laminated composite panel is developed using three-dimensional analysis including the super-element concept with reduced integration, large deformation analysis to handle buckied sublaminate and MVCCI for estimating strain energy release rate components at curved delamination front. Numerical results show that the delamination is critical when it occurs in the top (or bottom) few layers of a laminate. It is demonstrated that the studies presented can be used to determine delamination tolerance level from the safety point of view in design and operation phases of structural components.

\section{References}

Badari Narayana K. Dattaguru B, Ramamurthy T S, Vijayakumar K 1994 A general procedure for modified crack closure integral in 3D problems with cracks. Eng. Fracture Mech. 48: 167-176

Buchholz F G 1984 Improved formulae for the FE calculation of the strain energy release rate by the modified crack closure integral method. Proc., 4th World Congress and Exhibition in Finite Element Methods, Interlaken, pp 650-659

Broek D 1978 Elementary engineering fracture mechanics 2nd edn (Amsterdam: Sijthoff and Noordhoff) pp 74-82

De Georges D G, Maison S, Tallero D, Petitniot J L 1992 Buckling and post-buckling behavior of a delamination in a carbon-epoxy laminated structure: Experiments and modeling, debonding/delamination of composites. AGARD-CP-530, 7.1-7.11

Jones R, Callinan R, Teh K K. Brown K C 1984 Analysis of multi-layer laminates using threedimensional super-elements. Int. J. Numer. Methods Eng. 20: 583-587 
Naganarayana B P 1991 Consistency and correctness principles in quadratic displacements type finite elements. Ph D thesis, Aerospace Engineering Department, Indian Institute of Science, Bangalore

Raju I S 1986 Simple formulas for strain energy release rate with higher order and singular finite elements. NASA CR 178186

Raju I S, Crews J H Jr, Aminpour M A 1988 Convergence of strain energy release rate components for edge delaminated composite laminates. Eng. Fracture Mech. 30: 383-396 (also available NASA-TM 89135, 1987)

Rybicki F E, Kanninen M F 1977 A finite element calculation of stress intensity factors by modified crack closure integral. Eng. Fracture Mech. 9: 931-938

Whitcomb J D 1984 Strain energy release rate analysis of cyclic delamination growth in compressive loaded laminates: Effects of defects in composite materials. ASTM STP 836, pp 175-193, (also available as NASA-TM-84598, 1983)

Whitcomb J D 1988 Mechanics of instability related delamination growth. NASA-TM-100622

Williams M L 1959 The stress around a fault or crack in dissimilar media. Bull. Seismol. Soc. Am. 49: 199-204

Zienckiwicz O C, Taylor R L 1991 The finite element method 4th Int. edn (New Delhi: McGraw Hill) 2: $284-304$ 